Vallejos Yopán, Rosa; Amías Murayari, Rosa (2015). Diccionario Kukama-Kukamiria* Castellano. Lima: FORMABIAP y AIDESEP. Pp. 312. ISBN 2015-11522.

Resenhado por María C. Chavarría Mendoza (Universidad Nacional Mayor de San Marcos, Lima, Perú)

El Diccionario Kukama-Kukamiria * Castellano es un diccionario descriptivo, sincrónico de la lengua Kukama-Kukamiria de la familia Tupí Guaraní. El actual territorio de los hablantes de esta lengua se ubica en los departamentos de Loreto y Ucayali, en el Perú. La otra lengua de esta misma familia es Omagua. Según datos obtenidos por el Ministerio de Cultura (2015), la población de las comunidades del Pueblo Kukama-Kukamiria se estima en 20,511 personas, organizados en aproximadamente 120 comunidades.

La lengua Kukama-Kukamiria según el Documento Nacional de Lenguas Originarias del Perú (2013) está seriamente amenazada pero: “A diferencia de las demás lenguas que presentan alta vulnerabilidad, este grupo de lenguas está siendo revitalizado por decisión de sus propios hablantes" (p. 442). La UNESCO considera a esta lengua como idioma "seriamente en peligro" de extinción. En este contexto, una documentación lexicográfica es un intento de apuntalar su proceso de revitalización y mantenimiento.

La población kukama se ha mostrado muy activa en este proceso dirigido especialmente a los jóvenes y niños que ya no hablan la lengua. Los adultos y mayores, con mucho esfuerzo, y con el apoyo de sus sabios y sabias están difundiendo su lengua especialmente entre los jóvenes, a través de diversos medios, entre ellos la radio Ukamara. ${ }^{1}$ Además, han usado múltiples recursos, entre ellos los medios digitales, haciendo videoclips que se han hecho muy populares en las redes sociales. Recientemente, Radio Ukamara, en premio a su tarea de difundir la cultura kukama durante 10 años recibió el Premio Nacional como "Personalidad meritoria de la cultura 2015", otorgado por el Ministerio de Cultura del Perú.

Rosa Vallejos Yopán y Rosa Amías Murayari son coautoras de este importante trabajo publicado por el Programa de Formación de Maestros Bilingües de la Amazonía PeruanaFORMABIAP en 2015. Ambas han sido profesoras de este programa en distintos momentos de su vida profesional y han colaborado en producir textos educativos en la lengua. Vallejos es lingüista formada por la Universidad Nacional Mayor de San Marcos y obtuvo su Master y Doctorado en la Universidad de Oregon (Estados Unidos) con una tesis sobre la gramática de la lengua. Actualmente se desempeña como profesora de la Universidad de New Mexico. Es autora de una Gramática Kukama-Kukamiria (2016). ${ }^{2}$

Amías es natural de la comunidad Dos de Mayo, San Pablo de Tipishca, en el río Marañón y habla la variedad kukama. Además de ser miembro de la Federación ACODECOSPAT, se ha desempeñado como especialista del FORMABIAP y también conoce e identifica otras variedades, incluyendo el kukamiria. La señora Amías es una relatora reconocida de la tradición oral de su pueblo y también ha participado en la ilustración del Diccionario.

\footnotetext{
${ }^{1}$ radio-ukamara.blogspot.com

${ }^{2}$ Una reseña de la Gramática Kukama-Kukamiria será publicada en LIAMES 16(2): 2016.
} 
El Diccionario contiene aproximadamente 2,400 entradas léxicas y gramaticales. Muchas de estas entradas fueron recolectadas durante un trabajo de investigación previo (2004 y 2012) que se llevó a cabo en 16 comunidades. Esta primera investigación estaba orientada a recoger muestras significativas de la oralidad del Pueblo Kukama-Kukamiria (relatos, icaros, cantos, conversaciones) y contó con la colaboración de 42 hablantes.

El objetivo del Diccionario es contribuir a revitalizar la lengua Kukama-Kukamiria y en las primeras líneas introductorias se señala que presentan esta investigación lexicográfica "con la finalidad de acercar a nuestros lectores al mundo de los Kukama-Kukamiria". Es importante destacar que las autoras incluyen información y documentación fotográfica de los colaboradores del Pueblo Kukama-Kukamiria, entre los que se encuentran sabios y sabias indígenas, maestros bilingües y autoridades comunales. Este dato sobre los hablantes de quienes se ha recogido la información es pasado por alto, muchas veces, en publicaciones de este tipo y solo se suele mencionar a los revisores mas no a los hablantes. Otro hecho relevante del Diccionario es que la investigación en el campo ha recibido importantes subvenciones de organizaciones académicas de Estados Unidos, Inglaterra y Dinamarca, respectivamente. ${ }^{3}$ Esta información puede ser un dato interesante para quienes trabajan con lenguas que se encuentran en distintos estadios de "peligro" o vulnerabilidad, como es el caso de varias lenguas amazónicas y tienen en mente publicar un trabajo similar. Todo diccionario tiene un objetivo que debe ser explícito desde su comienzo. Según las propias autoras, el diccionario se hizo con el deseo de que sea de utilidad principalmente a los siguientes grupos de usuarios: (i) hablantes actuales: para ayudarlos a recordar información que puede estarse perdiendo (ii) jóvenes kukama-kukamiria que están aprendiendo su lengua originaria; (iii) maestros bilingües: para ayudarlos a enseñar la lengua a los niños y (iv) investigadores interesados en la Amazonía.

Estructura del diccionario. Está organizado en tres partes (I): Introducción: incluye la descripción del diccionario, las características de la lengua, iniciativas para revitalizar la lengua. (II): kukama-kukamiria/castellano: la parte central del diccionario y (III): castellano/kukama-kukamiria: índice o buscador en castellano.

\section{Introducción}

Después de los Agradecimientos, se hace una detallada descripción de las principales secciones de esta obra. Seguidamente, aparece una guía de uso que es muy didáctica, aunque omite mencionar que para toda consulta es necesario seguir un orden alfabético. Esto nos remite a otra información importante: el alfabeto de la lengua Kukama-Kukamiria, que fuera usado desde 1988 por FORMABIAP.

Los Componentes de las entradas lexicales incluyen:

${ }^{3}$ National Science Foundation, National Endowment for the Humanities y Hans Rausing Endangered Languages Project. La publicación corrió a cargo de Axis-Dinamarca. 
I. Entrada lexical en Kukama-Kukamiria. En el caso de un diccionario bilingüe de una lengua indígena-castellano, nuestras miradas van a cómo aparece, en primer lugar, la lengua indígena pues probablemente la escritura es reciente y proporcionar su transcripción fonética con la marca silábica es imprescindible. Lamentablemente este dato no aparece. La entrada léxica proviene de la variedad kukama hablada por una de las autoras.

\section{Variedad dialectal}

En principio, aparece la forma base kukama. Si hubiera otras, se incluye las otras variantes que se han podido documentar. Nos hubiera gustado encontrar una sección donde se explique en qué consiste la diferencia fonético-fonológica de unas y otras. Sin embargo, para los posibles lectores recomendamos la lectura de un trabajo previo de Vallejos (2007) y Valero (2015) allí se esclarece el porqué de las denominaciones Kukama-Kukamiria y aclara que no son dos hablas distintas. Sobre el origen de la autodenominación. Citamos:

Los términos kukama y kukamiria corresponden a lo que en la literatura lingüística y etnográfica podemos encontrar como cocama/kokama y cocamilla/kukamilla respectivamente, y son producto de la reflexión del personal indígena del FORMABIAP, quienes han hecho efectivo su derecho a autodenominarse (p. 91).

\section{Clase de palabra}

¿Qué tipo de palabra es? ¿Es un adjetivo o es un verbo? Esta información particularmente es útil sobre todo si en el futuro se hace una gramática pedagógica. Para indicar la clase de palabra se usan abreviaturas en color verde que van encerradas en paréntesis como $(N)$ para Nombre, verbo $(V)$. Pudo haberse escogido otro color porque prácticamente es difícil de leer.

Además de nombres, aparecen adverbios, demostrativos, pronombres, partículas, onomatopeyas y una información gramatical muy pertinente: morfemas ligados. En relación a la gramática de la lengua Kukama-Kukamiria, en las entradas lexicográficas se incluye tipos de verbos. Por ejemplo, si el verbo es estativo o transitivo. Creemos que si se ha incluido la información sobre el uso con ejemplos sencillos, las dudas pueden aclararse. Considerando que una de las autoras ha escrito una reciente gramática de la lengua (Vallejos 2010, 2016), la información sobre la función que cumple cada palabra hace que el diccionario sea realmente funcional.

\section{Glosa y/o definición en castellano}

La glosa en castellano, seguramente, ha significado un reto para las autoras pues presumimos que se trata de una variante del castellano regional amazónico. El estudio del castellano amazónico se encuentra muy descuidado por los lingüistas en el Perú, salvo escasas excepciones (Escobar 1978; Ramírez 2003). Para quienes hemos trabajado con 
lenguas amazónicas, un diccionario bilingüe permite acercarnos al castellano regional, cualquiera fuera sus variantes. Revisando los vocabularios de castellano amazonense hemos podido encontrar que muchas palabras provienen del kukama, especialmente en lo que se refiere al léxico de la fauna ictícola (Herrera 2003; Valero 2015) y de algunas especies maderables y frutas. Por ello, este Diccionario Kukama-Kukamiria significa un valioso aporte al estudio de los que se ha llamado "castellano amazónico" y que evidentemente no es uniforme, siendo la variante de Loreto y Ucayali la más conocida, pero no la única.

\section{Nombre científico}

Este dato es importante porque muchos nombres de animales o plantas no tienen su correlato en castellano y por ello es importante incluir el nombre científico. Esta información sirve especialmente además a los biólogos, ingenieros agrónomos, forestales, ecólogos, educadores y especialistas en educación ambiental. En la presentación que se hizo para el Diccionario, se hace hincapié en la importancia del conocimiento kukama sobre su medio ambiente: ${ }^{4}$

\footnotetext{
Este diccionario documenta muchos procesos y prácticas socio-culturales, y recoge buena parte del sofisticado conocimiento que poseen sobre su entorno natural. Por ejemplo, una de las actividades fundamentales en la vida de los kukama-kukamiria es la pesca. Su conocimiento profundo del mundo acuático se refleja en su lengua, la cual posee más de un centenar de nombres para referirse solo a diferentes especies de peces. También se han documentado más de 120 términos para nombrar diferentes especies de aves, 25 para especies de palmeras, entre otros.
}

Efectivamente, el Diccionario contiene información sobre la biodiversidad de una parte extensa de la Amazonía y por ello encontramos un repertorio muy rico vinculado al medio ambiente, no solo fauna y flora de distintos ecosistemas sino también su manejo. Anteriormente, FORMABIAP había publicado La vida secreta de las plantas medicinales en los pueblos Kichwa, Kukama-Kukamiria y Tikuna (2008). Y estos saberes son los que deben consignarse y escribirse. La megadiversidad de nuestro país coincide con una gran variedad de culturas y lenguas que hacen del Perú un país plurilingüe y multicultural. Es importante también deslindar que la necesidad de proteger esta megadiversidad del bosque conlleva el compromiso de preservar este saber de los pueblos indígenas. Esta preocupación aparece formulada en la legislación nacional e internacional. Y no solo proviene de las organizaciones orientadas a la conservación, proviene de los mismos indígenas. Y este es un hermoso resultado que observamos en el Diccionario. Ya la UNESCO había recomendado antes:

Los vínculos de entre lengua, cultura y medio ambiente sugieren que la diversidad biológica, cultural y lingüística debería estudiarse conjuntamente, como manifestaciones distintas, pero estrechamente relacionadas de la diversidad de la vida en la Tierra (Unesco Terra Lingua 2003: 7).

${ }^{4}$ http://www.formabiap.org/sitio/index.php/publicaciones/138formabiap-presento-los-libros-diccionariokukama-kukamiria-castellano-y-cuidando-nuestro-territorio 
$\mathrm{Y}$ este Diccionario es el hermoso resultado de haber trabajado teniendo en mente que los hablantes son los poseedores de un saber que va más allá del valor de una entrada lexical. Aquí tenemos un ejemplo:

\begin{abstract}
Aitseta Variante: aetseta. V. 'embrujar, hechizar, hacer daño. Según los kukama-kukamiria los que hacen daño son los brujos poderosos llamados payun, también los espíritus malos de algunos árboles como la madre de la lupuna colorada o de la catahua. Cuando nos hacen daño, uno se enferma y solo puede ser tratado por un curandero o sabio ikuan, por medio de dietas y vegetales... (p. 21).
\end{abstract}

\title{
6. Ejemplo
}

Los contextos de uso de estas palabras kukama son importantes y permiten esclarecer los casos de polisemia. Son introducidos después de dos barras. Los contextos son seguidos de una traducción al castellano. Ejemplo:

ariwi $V$. emerger, flotar, boyar $\|$ Ipira umanun ariwi ra puapuka 'El pescado muerto flota cuando está podrido." ||Tsiwiiwatsu ariwi tuyuka tsitsariwa 'La lombriz sapana sale a la superficie de la tierra' (p. 40).

\section{Campo semántico}

La necesidad de presentar las palabras organizadas de acuerdo al significado común que comparten ha hecho desarrollar los diccionarios por campos semánticos. Este no lo es, pero indica a qué campos semánticos pertenece la entrada lexicográfica. Por ejemplo, aquella relativa a la fauna: aves, peces u otra vinculada a la flora, por ejemplo: palmeras. También tiene árboles, lianas, mamíferos, insectos; pero además otros campos menos conocidos como enfermedades, onomatopeyas, etc. Una información social valiosa es aquella que da cuenta de apellidos, relaciones de parentesco y comunidades. Al ordenar las palabras solo por orden alfabético, parte de esta valiosa información se puede perder, pero al incluir campo semántico al final nos permite rescatarla en toda su esencia. Hemos encontrado que el apellido Inuma es kukama y también se encuentra extendido en Madre de Dios, al igual que Arimuya, por razones históricas que deben ser develadas.

\section{Enlace correferencial}

Aquí se remite a otras entradas que se hallan semánticamente emparentadas. Aparecen en color rojo y este es tan tenue que es difícil de leer, obviamente este es un problema de edición que se puede enmendar en una siguiente reedición. 


\section{Ilustración}

Es uno de los aspectos más relevantes de este Diccionario. La gran mayoría de diccionarios que se han hecho en el Perú adolecen de la parte gráfica, incluso los del Instituto Lingüístico de Verano. Felizmente, las autoras han recurrido a una reconocida autoridad ambiental como la Amazonía: Guía Ilustrada de Flora y Fauna, publicación del Ministerio del Ambiente, para sus entradas de fauna y flora. El diccionario contiene cerca de 400 apoyos visuales (entre dibujos y fotos) y de ellos, 54 dibujos que corresponden a la cultura material, conocimientos y procedimientos kukama-kukamiria fueron producidos por la coautora Rosa Amías Murayari.

\section{Sentido}

Cuando buscamos un diccionario bilingüe, esperamos que ofrezca la siguiente información: los diversos significados pues es un poco raro que una sola palabra tenga un solo significado. Parece que a ello se debe esta categoría que no hemos ubicado fácilmente en el diccionario.

\section{Pronunciación}

Si se trata de una lengua como el Kukama-Kukamiria que se encuentra en proceso de revitalización, una guía de la pronunciación es necesaria sino decirlo urgente pues las nuevas generaciones que desean aprender esta lengua de sus antepasados, necesitan saber cómo suena cada entrada léxica. Es cierto que no todos lo tienen, pero es lo deseable. El diccionario no presenta esta información para todas las entradas solo para algunas. Sería oportuno para una próxima edición incluir la forma fonética encorchetada y la notación silábica. Estos datos pueden ayudar en el proceso de adquisición de la escritura del Kukama-Kukamiria.

Luego el Diccionario presenta el alfabeto usado por FORMABIAP desde 1988:

$<a, c h, e, i, \dot{i}, j, k, m, n, p, r, s h, t, t s, u, w, y>$

Los Diccionarios suelen presentar frases idiomáticas que aquí aparecen bajo el título de Algunas expresiones frecuentes, donde se incluyen ciertas construcciones para expresar sentimientos o emociones que se crean teniendo como base una parte muy importante del cuerpo humano: el corazón. Luego aparecen algunos saludos y el contexto en que pueden ser usados.

Aunque no hay una descripción gramatical, se ha insertado una sección en la Introducción denominada Clases de Palabras donde se señala que el diccionario contiene raíces, "algunas bases derivadas y morfemas gramaticales" (p. 12). Sigue una presentación de cómo se producen formas derivadas con algunos ejemplos. Se incluye también una descripción de la creación de palabras mediante composición, donde se precisa que es altamente funcional para los nombres de plantas y animales. Se escriben separadas si cada una tiene un acento. 
El acento. No se marca porque es altamente predecible. Si es necesario hacerlo se coloca entre corchetes, por ejemplo: pua [puá] o para desambiguar el siguiente par mínimo: kunia [kúnia] 'hermana de hombre' y kuníya [ kunía] 'prima de hombre'.

Discurso de mujeres versus discurso de hombres. Una de las características que distingue a esta lengua es la diferencia que se establece entre el habla masculina y femenina en algunas categorías gramaticales. No se conoce otra lengua peruana que tenga esta característica. Así lo señalan las autoras del Diccionario:

Es decir, en kukama-kukamiria la categoría gramatical género no hace referencia al objeto del que se habla (como en el caso del castellano) sino al género biológico del hablante. (...). Esta distinción de enero se refleja en los pronombres personales, pronombres demostrativos, marcadores de plural y en conectores oracionales y discursivos (p. 14).

Después de mostrar un cuadro que ejemplifica las características del discurso de mujeres y hombres, sigue una breve referencia a los Morfemas ligados que incluye los sufijos y clíticos, finalizando de esta manera la sección gramatical.

La Introducción termina con El pueblo Kukama-Kukamiria y los esfuerzos para preservar su lengua, interesante reseña sobre el proceso de revitalización de la lengua y los diversos agentes sociales que se han involucrado en esta tarea.

II. Kukama-Kukamiria * Castellano. Es el Diccionario mismo que hemos ido describiendo en su estructura básica y los elementos que aparecen en cada entrada léxica.

III. Castellano-Kukama-Kukamiria ocupa solo una pequeña parte del volumen (pp. 276-311) pues las entradas se presentan a tres columnas sin mayores descripciones.

Este no es el único Diccionario sobre esta lengua, hubo uno antes denominado Breve Diccionario Analítico Castellano-Tupi del Perú. Sección Cocama (1988) del P. Lucas Espinoza. Han pasado casi 30 años desde que esta obra que fuera dejada como manuscrito por este misionero agustino se publicara en Iquitos bajo los auspicios de CETA, con un prólogo del lingüista Ángel Corbera Mori, quien también revisara el manuscrito con la ayuda del P. Luciano Silva y la señora Sofía Pacaya. Escritos en épocas distintas, estas dos piezas lexicográficas sin duda dejan un claro mensaje: la lengua Kukama-Kukamiria vive.

\section{$\overline{\text { Referencias }}$}

Escobar, Alberto (1978). Variaciones sociolingüísticas del castellano en el Perú. Lima, Peru: Instituto de Estudios Peruanos.

Espinoza, Lucas (1989). Breve diccionario analítico Castellano-Tupi del Perú. Sección CocAMA. Iquitos: CETA.

FORMABIAP (2008). La vida secreta de las plantas medicinales en los pueblos Kichwa, Kukama-Kukamiria y Tikuna. Iquitos: FORMABIAP.

Herrera, Enrique (2003). Etnografia del aprovechamiento de la fauna ictícola en una comunidad Ese Ejja de reciente sedentarización. La Paz: Universidad Mayor de San Andrés. (Tesis de licenciatura.) 
MINAM (2010). Amazonia: Guía ilustrada de flora y fauna. Lima: Ministerio del Ambiente.

MINEDU (2012). Documento nacional de lenguas originarias del Perú. Lima: Ministerio de Educación del Perú.

Ramírez, Luis H. (2003 [2013]). El español amazónico hablado en el Perú. Lima, Perú: Juan Gutemberg Editores.

UNESCO (2003). Compartir un mundo de diferencias: La diversidad lingüistica, cultural y biológica de la Tierra. Barcelona: Centro UNESCO de Cataluña.

Valera, Gildo Martín (2015). Clasificación y saberes de la piscifauna en el pueblo Ese Eja (Takana). Lima: Universidad Nacional Mayor de San Marcos. (Tesis para optar el Grado de Bachiller.)

Vallejos Yopán, Rosa (2007). Fonología de la variedad Kukama-Kukamiria del río Huallaga. En Fonología, volumen I. Serie Descripciones Lingüísticas. Iquitos: FoRMABIAP.

Vallejos Yopán, Rosa (2010). A grammar of Kokama. Eugene: University of Oregon. (Ph.D. dissertation.)

Vallejos Yopán, Rosa (2016). A grammar of Kukama-Kukamiria: A language from de Amazon. Leiden: Brill's Studies in the Indigenous Languages of the Americas.

Recebido: 9/6/2016

Versão corrigida: 12/6/2016

Aceito: 15/6/2016 